\title{
Puntos de inflexión y recuperación del desarrollo infantil tras experiencias iniciales de adversidad
}

\section{Turning points and developmental recovery after adverse early childhood experiences}

\author{
Alicia Muñoz Silva \\ Departamento de Psicología Evolutiva y de la Educación, Universidad de Huelva, España.
}

Disponible online 30 de abril de 2012

\begin{abstract}
El presente trabajo analiza la capacidad de recuperación del desarrollo infantil a partir de la revisión de los resultados obtenidos por diferentes investigaciones que han examinado las trayectorias evolutivas de niños y niñas con experiencias iniciales muy adversas pero que, gracias a factores de diversa índole, pudieron experimentar un punto de inflexión en sus vidas. Tras la exposición de los estudios y sus principales hallazgos, se reflexiona sobre su contribución a la compresión de las posibilidades de recuperación del desarrollo infantil y el alcance y limitaciones de la intervención con niños y niñas expuestos a situaciones de riesgo o adversidad.

Palabras clave: Puntos de inflexión; Adversidad; Recuperación; Desarrollo Infantil.

The aim of this article was to analyse the potential for developmental recovery based on a review of different studies on the development of children who, despite early adverse experiences, had experienced a turning point in their lives due to various kinds of events. The implications of the research and findings are discussed in relation to understanding the recovery of child development and the possibilities for interventions with children exposed to risk or adversity.
\end{abstract}

Key words: Turning points; Adversity; Recovery; Child Development.

Correspondencia: Alicia Muñoz Silva. Departamento de Psicología Evolutiva y de la Educación. Campus de El Carmen. Avenida de las Fuerzas Armadas, S/N. 21071 Huelva. E-mail: amsilva@uhu.es 
Muchos niños y niñas nacen y crecen en contextos que desde la psicología evolutiva podemos calificar como poco favorables para su desarrollo. Pero, afortunadamente, buena parte de ellos consiguen hacer frente a los riesgos de un modo exitoso, presentando un adecuado desarrollo a pesar de los evidentes obstáculos que se presentaban para el mismo. Se dice de estos niños y niñas que muestran lo que se denomina resiliencia, o lo que es lo mismo, una adaptación positiva -generalmente entendida como el afrontamiento adecuado de las tareas del desarrollo típicas de una determinada etapa- a pesar de experiencias de significativa adversidad o trauma, consideradas circunstancias de riesgo al asociarse con una alta probabilidad de ajuste negativo (Lemos, 2003; Luthar, 2006; Luthar, Cicchetti y Becker, 2000; Masten, 2001; Rutter, 2000; Vera, Carbelo y Vecina, 2006).

En la línea de los estudios sobre resiliencia ante la adversidad, el objetivo del presente artículo es analizar las posibilidades de recuperación del desarrollo de niños y niñas expuestos a circunstancias iniciales muy negativas pero que, por diferentes factores, experimentan un cambio sustancial en sus vidas, en virtud del que se amplían notablemente las oportunidades para un desarrollo más favorable. Entendemos que el artículo puede realizar una aportación significativa en este campo, ya que, sin duda, la reflexión sobre la evolución de las trayectorias vitales de estos niños y niñas contribuye a una mejor comprensión de la capacidad de recuperación del desarrollo y de las posibilidades y limitaciones de la intervención con niños y niñas en situación de riesgo o adversidad.

\section{Niños y niñas criados en instituciones con graves carencias físicas y psicoafectivas}

Los experimentos naturales en los que los niños y niñas son víctimas de ambientes muy pobres en sus primeros meses o años de vida, pero tiempo después comienzan a recibir un cuidado más sensible y estimulante, desgraciadamente nos proporcionan una oportunidad única para investigar hasta qué punto la infancia es un periodo sensible, la reversibilidad o irreversibilidad de las experiencias que en ella se sitúan y las posibilidades de recuperación de los efectos de las mismas.

Uno de los primeros y más importantes estudios al respecto fue el realizado por Dennis y sus colaboradores (Dennis, 1973, Sayegh y Dennis, 1965) con niños y niñas del hospicio libanés de Créche. Como consecuencia de la deprivación estimular predominante en la institución, los niños presentaban un severo retraso tanto a nivel psicomotor como cognitivo y lingüístico. Estos niños permanecían tumbados boca arriba en sus cunas durante buena parte de sus dos primeros años de vida, sin recibir prácticamente ninguna atención individual de sus cuidadores. Con un año no se sentaban y a los dos años de edad muchos de ellos no podían andar. También su desarrollo intelectual se retrasaba, pasando de un nivel medio en sus primeros meses a un CI alrededor de 50 puntos al año de edad. Sin embargo, y a pesar de las dificultades que presentaban estos pequeños, su desarrollo experimentó un progreso muy significativo tras una intervención aparentemente mínima desarrollada por estos investigadores. Dicha intervención consistió en que durante una hora diaria y a lo largo de quince días se sentó a los bebés con apoyo, animándoles a manipular juguetes muy simples (Sayegh y Dennis, 1965).

En el seguimiento longitudinal del desarrollo cognitivo de estos menores se observaron importantes diferencias de género, relacionadas con el hecho de que hasta los seis años de edad niños y niñas vivían juntos en la misma institución, pero a partir de ese momento pasaban a vivir en dos centros distintos, Zouk para las niñas y Brumana para los niños, este último con mejores condiciones estimulares que el de las niñas. Desde que se produjo el cambio de institución los chicos fueron aumentando gradualmente su CI hasta alcanzar a los 16 años una media de 80 , mientras que las niñas seguían manteniendo un promedio de 50 (Dennis, 1973).

\section{Pobreza y desnutrición infantil}

Otras situaciones menos extremas, como el desarrollo infantil en situaciones familiares de pobreza y/o con graves carencias de una estimulación rica y variada también pueden derivar en retrasos psicomotores, sociales o cognitivos. En este sentido, diversos estudios han focalizado su atención en las implicaciones para el desarrollo infantil de una de las consecuencias más directas de la pobreza, la desnutrición. Una investigación paradigmática en este ámbito es el trabajo de Brown y Pollit (1996), resumen de varias décadas de investigación (Pollit, 1987) destinadas a desentrañar todas las posibles influencias de la desnutrición en el desarrollo infantil y los beneficios de intervenciones centradas en mejoras nutricionales.

Los resultados de la investigación de Brown y Pollit (1996) con niños y niñas que vivían con sus familias en aldeas guatemaltecas muestran que la malnutrición o desnutrición pueden influir en diversos aspectos del desarrollo, pues no sólo se afecta la ganancia de peso, se retrasa el desarrollo físico y psicomotor y se disminuye la resistencia a las infecciones, sino que también pueden resultar alterados los procesos cognitivos. Dicha influencia no se debe únicamente a que la malnutrición tiene el potencial de producir daños permanentes en el cerebro, principalmente en los casos de desnutrición grave, sino también a que las deficiencias nutricionales suelen interactuar con otras carencias derivadas de vivir en condiciones de pobreza, tales como dificultades económicas, escasa asistencia médica, pocas oportunidades educativas, un ambiente poco estimulante o una pobre interacción de los niños y niñas con su entorno en parte debida a la falta de energía vital. La desnutrición también limita el aprendizaje, ya que la capacidad de aprender tiene que ver con el tiempo que hace que se ha comido, con lo que el desayuno diario antes de ir a la escuela es muy importante, sobre todo para los niños desnutridos. Sin embargo, e incluso cuando existe daño cerebral debido a la malnutrición en los dos primeros años de vida, parte del daño puede ser reversible. Destacan 
además estos autores que es erróneo restringir la atención a los dos primeros años de vida pues también puede resultar afectado el cerebro por la desnutrición después de esta edad.

La intervención desarrollada con una muestra de estas familias guatemaltecas reveló que un elemento clave para el desarrollo es la mejora de la nutrición temprana, que se mostraba aún más importante cuanto más negativas eran las demás condiciones socioeconómicas. Así, los niños que más se beneficiaron de tomar suplementos alimenticios eran los que pertenecían a las familias más pobres de las aldeas. El efecto de los suplementos nutricionales perduró aún después de que se acabase el programa, y se debió en buena medida a que la mejora alimenticia contribuyó a una mejor tasa de crecimiento, capacidad motora, recuperación de enfermedades y desarrollo social y emocional. De este modo, las mayores posibilidades de explorar el entorno físico y social mejoraron las habilidades cognitivas. Pero estos autores también advierten que las relaciones entre desnutrición y aprendizaje son bastante complejas y pueden estar mediadas por otros factores. Por ejemplo, a los niños con menor crecimiento para su edad se les puede tratar de una forma más infantil, estimulándolos y exigiéndoles menos; tampoco se puede desdeñar que los cerebros de los niños peor alimentados no tengan también cierto grado de deterioro que dificulte su rendimiento (Brown y Pollit, 1996).

No obstante, la mejora en la alimentación no pudo compensar todos los efectos negativos de la pobreza, pues aun en el mejor de los casos, los resultados intelectuales de los niños y niñas participantes en el estudio no alcanzaban los obtenidos por niños de clase media de otras zonas de Guatemala. Por ello, Brown y Pollit (1996) subrayaron la necesidad de que las mejoras nutricionales se insertasen en el seno de programas más integrales y fuesen acompañadas por medidas de asesoramiento a los padres sobre la crianza y el desarrollo de los hijos, de la estimulación en los niños y niñas de las interacciones sociales y con su entorno físico, y también de progresos en la atención médica y la educación, con programas de intensificación y educación compensatoria de posibles carencias.

Otro trabajo en esta misma línea es la investigación de Colombo, de la Parra y López (1992), en la que, siguiendo el enfoque global iniciado por Pollit (1987), se muestran las repercusiones físicas e intelectuales de haber sufrido desnutrición en la primera infancia. Para ello, se estudió a un grupo de escolares chilenos que fueron hospitalizados y tratados por malnutrición en sus primeros dos años de vida. Posteriormente, algunos de estos niños y niñas volvieron con sus familias de origen -que, aunque participaron en un programa para mejorar la crianza y estimulación de sus hijos, siguieron teniendo un nivel socioeconómico bajo-, otros fueron adoptados por familias de nivel socioeconómico medio o alto, y otro grupo pasó a vivir en instituciones. Los resultados más importantes revelan que, a pesar de que niños y niñas alcanzaron después de su tratamiento hospitalario similares niveles de desarrollo físico y psicológico, cuando fueron estudiados en los comienzos de la etapa primaria el desarrollo de los integrantes de los tres grupos difería notablemente. Así, los niños y niñas adoptados obtuvieron mejores resultados tanto en las medidas de desarrollo físico como de desarrollo intelectual, resultados que además se encontraban dentro de parámetros de absoluta normalidad. Por el contrario, los niños institucionalizados eran los que presentaban peores niveles de desarrollo en ambos aspectos, quedando los niños y niñas que habían continuado con sus familias de origen a medio camino entre unos y otros. Las conclusiones que estas investigadoras extraen de su estudio subrayan, al igual que el estudio de Brown y Pollit (1996), la posibilidad de revertir los efectos para el desarrollo de un período temprano de desnutrición severa, pero siempre que los programas de recuperación se enfoquen de un modo global, considerando no solo la necesidad de mejoras nutricionales, sino también de que niños y niñas crezcan en un ambiente que les proporcione de modo estable las interacciones y la estimulación que necesitan, tanto para su desarrollo físico como psicológico. A juzgar por los resultados de la investigación, estas oportunidades eran proporcionadas por las familias adoptivas del estudio, pero no por las instituciones ni las familias de origen de estos menores, que no podían satisfacer de modo óptimo las necesidades físicas y educativas de estos niños y niñas.

\section{La adopción como punto de inflexión}

Pero, sin duda, y como ha puesto de relieve el último de los estudios revisados en el apartado anterior, la mayoría de las investigaciones que analizan puntos de inflexión en el desarrollo infantil tras experiencias iniciales de adversidad se centran en el análisis de la evolución de niños y niñas tras la adopción, ya que esta introduce en las experiencias vitales infantiles una profunda discontinuidad, un marcado contraste entre las experiencias previas y las posteriores a la adopción (Palacios, 2007). Entendiendo que esta discontinuidad tiene que ver con una transición hacia contextos de desarrollo más positivos para el desarrollo infantil, la adopción es considerada por muchos autores como una medida de protección a la infancia que ofrece a niños y niñas que han crecido en contextos de adversidad grandes oportunidades para superar los problemas, retrasos y dificultades que han experimentado en su desarrollo evolutivo (Colombo et al., 1992; González, Quintana, Fernández, Linero, 2003; Juffer, van Ijzendoorn y Palacios, 2011; Rutter et al., 2002).

Comenzamos nuestra revisión con el anteriormente citado estudio de Dennis y sus colaboradores (Dennis, 1973; Sayegh y Dennis, 1965), que además de la evolución de los niños institucionalizados también analizó la recuperación de los menores que pudieron ser adoptados, ya que en 1956 la adopción quedó legalizada en El Líbano y muchos de los niños y niñas de Créche fueron a vivir con familias libanesas o americanas. Los principales resultados del seguimiento de estos niños y niñas revelaron que, cuando la adopción tuvo lugar antes de los dos años de edad, los niños progresaron rápidamente en su desarro- 
llo intelectual, alcanzando a los 4 años un CI medio de 100. Si la adopción tenía lugar después de los dos años, la recuperación no era tan completa, siendo menor cuanto mayor era la edad de los niños en el momento de la misma.

Continuando con el cambio en la trayectoria vital que supone la adopción, la investigación de Rutter y colaboradores (O'Connor, Rutter, Beckett, Keaveney y Kreppner, 2000; Rutter y The English and Romanian Adoptees Study Team, 1998; Rutter et al. 2002) es considerada uno de los principales estudios sobre las posibles secuelas a largo plazo de las privaciones iniciales generalizadas. Rutter y colaboradores realizaron un seguimiento de 165 niñas y niños rumanos, la mayoría criados en instituciones de muy baja calidad y con serias privaciones psicológicas, que posteriormente fueron adoptados en Gran Bretaña por familias con un nivel socioeconómico medioalto. En estrecha relación con sus condiciones de desarrollo, los niños presentaban severos retrasos físicos y problemas de salud, así como importantes dificultades cognitivas, sociales y emocionales.

Se realizó un seguimiento longitudinal cuando los niños tenían 4 y/o 6 años, cuyos resultados fueron comparados con los obtenidos por 52 niños ingleses adoptados antes de los 6 meses de vida, pero que no habían sufrido privaciones. En cuanto al desarrollo físico, cuando los niños rumanos alcanzaron los 4 años la recuperación conseguida en el peso y la altura fue espectacular, aunque la media era ligeramente inferior a la de la población general británica. Los progresos en su desarrollo psicológico también fueron notables, aunque con importantes diferencias interindividuales. Respecto al desarrollo cognitivo, los resultados indican que cuando los niños habían sido adoptados antes de los 6 meses la recuperación de las dificultades era casi total a los 4 años, lo que no ocurría cuando habían dejado las instituciones con más edad y particularmente con más de 2 años.

De todos los posibles factores influyentes de las condiciones de vida en estas instituciones -incluyendo la malnutrición, las enfermedades o el abuso de drogas psicotrópicas-, Rutter y su equipo destacan el papel de la falta de atención individual prestada a los niños. Además, el tiempo de permanencia en la institución se destacó más importante para el desarrollo cognitivo que el tiempo en la familia adoptiva. Estos autores también resaltan que los procesos de recuperación no se limitaban a los primeros meses de vida en la familia adoptiva, sino que eran mucho más extensos, particularmente en los niños con más dificultades.

En relación al desarrollo social, el comportamiento de los niños era bastante normal a los 4 y 6 años, pero cuanto mayor era la edad a la que habían sido adoptados se observó un menor juego simbólico y de roles y más conductas de búsqueda indiscriminada de contacto social. Aunque con menor grado, estas dificultades se observaron incluso en niños que habían sido adoptados antes de los 6 meses. Un tercio de los niños adoptados con más de 6 meses tenían además problemas de atención, inquietud, impulsividad e hiperactividad. Los rasgos autistas también eran más frecuentes. El estudio también constata que, incluso para los niños y niñas rumanos adoptados con menos de 6 meses, la probabilidad de presentar problemas en su desarrollo era mayor que para los menores de origen británico, lo que se explicaría con el hecho de que estos niños y niñas no habían sufrido privaciones en sus primeros meses de vida.

La mayoría de estos menores pudieron ser nuevamente evaluados en los comienzos de la adolescencia. Un estudio centrado en su desarrollo cognitivo reveló que, además de la heterogeneidad que presentaba la evolución de los menores, los datos indicaban que los niños y niñas con más bajas puntuaciones en desarrollo cognitivo continuaron mejorando de los 6 a los 11 años. Al margen de esta mejora para los que presentaban un mayor retraso a los 6 años, persistía la diferenciación entre aquellos que habían sido adoptados antes de los 6 meses -quienes no mostraban secuelas de la adversidad inicial- y los que fueron dados en adopción con posterioridad a esta edad, que incluso experimentaron una bajada en sus puntuaciones, que era independiente de que hubiesen sido adoptados un poco más tarde de los 6 meses o más cerca de los 42 meses, edad máxima a la que se produjo la adopción (Beckett et al., 2006). Otros estudios se centraron principalmente en el análisis del tipo de apego desarrollado por los niños o la presencia de problemas emocionales o conductuales, mostrando de nuevo cómo los niños que permanecieron en las instituciones después de los 6 meses de edad tenían una probabilidad mucho más alta de acusar problemas en estos ámbitos que aquellos que pudieron salir de estas instituciones a más temprana edad (Kreppner et al., 2007).

Entre las áreas menos estudiadas en relación a la reversibilidad de los efectos de carencias tempranas se encuentra el desarrollo lingüístico. En un claro intento de compensar esta laguna, González et al. (2001) desarrollaron una investigación con la finalidad de analizar el nivel de lenguaje de un grupo de niños en acogimiento preadoptivo en dos momentos: justo antes de comenzar a vivir con su nueva familia y al menos un año después del comienzo de la medida de protección. Las interesantes conclusiones del estudio dan respuesta a las dos principales preguntas que nos hacemos en el presente trabajo: cuáles son las posibles repercusiones de experiencias iniciales adversas y qué posibilidades y en qué condiciones se puede recuperar el desarrollo infantil de tales experiencias de adversidad.

En cuanto a la primera cuestión, las autoras hallaron que la mayoría de los niños y niñas estudiados mostraron un desarrollo lingüístico muy pobre, con afectación de todos los planos del lenguaje -forma, contenido y uso-, lo que se relaciona con las precarias condiciones en las que habían vivido durante bastantes años en sus familias de origen y en las que en muchos casos habían experimentado abandono y/o maltrato. Debemos también apuntar que todos estos niños y niñas habían vivido un tiempo en instituciones antes de su acogimiento preadoptivo, que en algunos casos abarcaba varios años de sus vidas. 
Por otra parte, y con las debidas cautelas, dado el tamaño y heterogeneidad de la muestra analizada, los resultados del estudio indican claras mejoras en los niveles lingüísticos de la mayoría de los niños y niñas después de haber pasado un tiempo en acogimiento preadoptivo, que oscilaba entre uno y dos años, lo que habla a favor de la capacidad de recuperación del desarrollo lingüístico infantil cuando mejoran las oportunidades para un desarrollo más óptimo, como suele suceder con la adopción. A pesar de estos progresos, para una buena parte de este grupo de niños todavía quedaba bastante para llegar a alcanzar las habilidades lingüísticas acordes a su edad, lo que las autoras relacionan con el escaso tiempo transcurrido desde su acogimiento preadoptivo en comparación con la duración de las carencias experimentadas por estos menores.

Los datos de otras investigaciones sobre el desarrollo de niños y niñas adoptados (Brodzinsky y Pinderhuges, 2002; Colombo et al., 1992; Duyme, Dumaret y Tomkiewicz, 1999; Fuentes y Fernández, 2001; González et al., 2003; Palacios y Sánchez, 1996; Palacios, Sánchez y Sánchez, 1997) coinciden en destacar que la mayoría de los niños y las niñas que han crecido en situaciones muy problemáticas pueden superar las consecuencias de estas experiencias si sus circunstancias contextuales cambian, aunque su ajuste se muestra asociado a diversas dimensiones, relacionadas tanto con los progenitores adoptivos como con los niños y las niñas adoptados. En este sentido, un resultado coincidente en varios estudios (Brodzinsky y Pinderhuges, 2002; Fernández y Fuentes, 2001; Fuentes y Fernández, 2001; Palacios et al., 1997) es señalar como factor de riesgo la edad de los niños en el momento de la adopción, debido no tanto a la edad en sí misma, sino a que supone un índice de la amplitud temporal en la exposición a circunstancias previas negativas. Por otra parte, la capacidad de recuperación del desarrollo de niños y niñas adoptados se muestra de modo mucho más evidente si, en vez de comparar a los niños adoptados con sus compañeros actuales, lo hacemos con niños que continúan viviendo en circunstancias familiares parecidas a las que ellos tuvieron en su pasado o con el desarrollo de niños y niñas institucionalizados (Juffer et al., 2011; Palacios, 1998; Palacios y Sánchez, 1996; Palacios et al., 1997; Roy, Rutter y Pickes, 2000).

En nuestro país, la investigación de Palacios, Sánchez y León (2005) sobre el desarrollo de niños y niñas procedentes de adopción internacional viene a confirmar, por un lado, la enorme capacidad de recuperación del desarrollo infantil, y, por otro, el peso de la edad a la que se produce la adopción. Los niños y niñas habían estado expuestos a circunstancias muy negativas: muchos de ellos vivieron algún tiempo con sus padres $u$ otras personas, siendo en ocasiones maltratados, para después ser abandonados. Posteriormente, la mayoría pasó a vivir en instituciones de muy baja calidad que no atendían adecuadamente sus necesidades de estimulación y de interacción privilegiada. Debido a ello, a su llegada a España un $40 \%$ estaba por debajo del percentil 3 en peso y altura, y un 30\% adicional por debajo del percentil 25 . Además, más del $60 \%$ tenían retrasos evolutivos (que en el 52\% de los casos eran graves) y un 56\% dificultades en el apego (graves en el 16\% de los casos).

Unos tres años después, niños y niñas habían hecho grandes avances hacia la normalidad, lo que dice mucho de su potencial de recuperación y también de las capacidades educativas de sus familias adoptivas. Al igual que sucedió en el estudio de Rutter y sus colaboradores, la recuperación de los niños y niñas estudiados por Palacios et al. (2005) resultó más completa en el plano físico que en el psicológico. Así, en el ámbito intelectual, una cuarta parte de los niños estudiados con menos de 6 años tenían retrasos evolutivos importantes; la tercera parte de los estudiados por encima de esa edad mostraba serias limitaciones intelectuales. Otro grupo de niños y niñas también acusaba problemas de conducta -impulsividad, hiperactividad, agresividad- y problemas en las relaciones de amistad, derivados de los trastornos iniciales del apego, aunque es obvio que las posibilidades de recuperación no estaban aún cerradas y los niños podían tener progresos posteriores en su desarrollo.

En cuanto a los elementos predictores de una mayor o menor recuperación, Palacios et al. (2005) destacan el papel de la edad y de las condiciones de la institución de partida. Así, en primer lugar, los datos del estudio muestran que, a mayor edad de los niños en el momento de la adopción, mayor probabilidad de problemas y desajustes en todos los ámbitos. Ello se explicaría no por la edad cronológica en sí misma sino por el hecho de que los niños tienen más posibilidades de vivir experiencias adversas, destacando entre ellas una más larga estancia en condiciones institucionales poco deseables. En el caso de la adopción internacional, a todo lo anterior se sumaría el hecho de que los niños deben adaptarse a una nueva cultura y/o idioma, lo que resultaría más complicado para los niños más mayores. Por otra parte, la mayor adversidad inicial también tuvo un gran peso, pues las condiciones de las instituciones rumanas eran las peores, estando estos niños entre los que tenían más problemas (Román, 2004). Sin embargo, aún en circunstancias aparentemente comparables, las trayectorias de los niños y niñas podían ser muy diferentes, mostrándose algunos de ellos especialmente resistentes a la adversidad inicial.

En nuestra revisión mencionamos en último lugar los estudios meta-analíticos llevados a cabo por Juffer y Van Ijzendoorn (Juffer et al., 2011) sobre más de 270 estudios centrados en la recuperación de niños y niñas tras la adopción. Estos análisis muestran que, en general, los niños y niñas adoptados experimentan una gran capacidad de recuperación en todos los aspectos de su desarrollo, superando ampliamente en todos los parámetros a niños y niñas institucionalizados, aunque se constatan algunos retrasos o dificultades cuando los niños y niñas son comparados con sus compañeros actuales. Estos retrasos o dificultades tienden a ser relativamente pequeños en áreas como los problemas de conducta y algo mayores en otras como el apego o el rendimiento escolar. Por otra parte, un dato muy significativo de los meta-análisis efectuados es que no se han 
hallado diferencias significativas entre niños adoptados y sus compañeros actuales en áreas como el peso, la altura, la inteligencia o la autoestima. En relación al papel de la edad de adopción, los datos indican que cuanto mayor era la edad los retrasos o dificultades estaban presentes con una mayor probabilidad.

\section{Reflexiones finales}

Al hacer un balance de los hallazgos de los diferentes estudios revisados debemos tener en cuenta que la complejidad del tema nos obliga a ser cautos en la interpretación de los mismos y en las conclusiones que de ellos podemos extraer. Ello no es sorprendente si atendemos al hecho de que el análisis entra de lleno en uno de los mayores interrogantes de la psicología evolutiva, la reversibilidad o irreversibilidad del desarrollo temprano, que además ha sido objeto de un importante debate desde los comienzos de la disciplina.

Sin embargo, no por ello debemos renunciar a apuntar lo que nos parece la aportación más importante que se desprende de la revisión de estos estudios que, desde prismas muy diversos, han realizado contribuciones de gran calado de cara a la comprensión de la protección y el riesgo para el desarrollo infantil. Así, entendemos que lo más sobresaliente de los estudios revisados es que pueden aportar a los profesionales de la intervención con niños y niñas en situación de riesgo una visión de las posibilidades y limitaciones que pueden tener sus intervenciones, además de un acercamiento a los recursos necesarios para afrontar de un modo más eficaz los innumerables retos de las mismas.

En este sentido, en primer lugar, los diferentes estudios vienen a reafirmar la idea de que las posibilidades de recuperación del desarrollo infantil dependen en buena medida de la conjunción entre la intensidad y la duración de los problemas o circunstancias ambientales que han podido afectar al desarrollo temprano, de modo que cuanto más adversas, pero también, prolongadas, hayan sido las circunstancias que rodeen al desarrollo inicial, la recuperación posterior probablemente será menos completa.

Lo anterior se torna especialmente preocupante si tenemos en cuenta que un dato recurrente en las investigaciones revisadas es que, en la mayoría de las ocasiones, las situaciones de deprivación física y psicológica suelen darse unidas y afectar preferentemente a niños, adolescentes y adultos que viven en condiciones socioeconómicas desfavorecidas. Por ello, no resulta sorprendente que los efectos de estas condiciones no se limiten a un solo plano del desarrollo, sino que se extiendan a diversas áreas del mismo.

Por otra parte, si los estudios en torno a la adopción coinciden en que la edad a la que se produce este punto de inflexión en la vida de niños y niñas es importante, es lógico preguntarnos si existe una edad límite, un periodo crítico fuera del que el desarrollo se puede ver seriamente amenazado. La revisión efectuada por Juffer et al. (2011) no encuentra una edad mágica (Palacios et al., 2005), antes de la cual sean casi inexistentes las secuelas de la adversidad inicial -que en los estudios de Rutter y sus colaboradores parece situarse alrededor de los 6 meses de edad- sino una tendencia continuada en la que una mayor edad implica un mayor riesgo de problemas. Si bien este dato parece ir en contra de la existencia de momentos críticos, sí que parece indicar que existen períodos especialmente sensibles para el desarrollo por parte de niños y niñas de determinadas capacidades, que deben ser especialmente aprovechados y tenidos en cuenta por parte de los profesionales de la protección a la infancia.

A partir de lo anterior, podemos afirmar que todas las evidencias ponen de manifiesto la relevancia no tanto de las experiencias tempranas puntuales, sino más bien de su incidencia continua y de la acumulación de sus efectos. De este modo, muchos niños y niñas criados durante la primera infancia en ambientes desfavorecidos continúan siendo afectados por condiciones de desventaja durante el resto de su infancia y adolescencia. Para muchos autores (Palacios, 1999; Rutter, 2000; Sameroff, 1998; Sameroff, Seifer, Baldwin y Baldwin, 1993) en la permanencia y efecto continuado y acumulativo de circunstancias de riesgo para el desarrollo está la clave para poder entender las dificultades cognitivas, emocionales, sociales y conductuales de muchos niños y adolescentes que se desarrollan en tales contextos. Afortunadamente, los resultados de los diferentes estudios revisados también muestran con gran contundencia que si ese ciclo de continuidad se rompe, si a los niños, niñas, y añadiríamos a sus familias, se les ofrece una oportunidad para un desarrollo más adecuado, es posible revertir al menos en parte, y en muchos casos de modo muy notable, el efecto negativo de la exposición a la adversidad. En estos casos, y tal y como se analiza con detalle en la revisión de Ramey y Ramey (1998), los programas de intervención que reúnen ciertos criterios (entre los que se destacan el comienzo temprano y la duración prolongada, la planificación, el cuidadoso seguimiento y el ser implementados por servicios de calidad) han demostrado ser muy eficaces para promover y mejorar el desarrollo infantil.

\section{Referencias}

1. Beckett, C., Maughan, B., Rutter, M., Castle, J., Colvert, E., Groothues, C., Kreppner, J., Stevens, S., O’Connor, T. G. y Sonuga-Barke, E. J. S. (2006). Do the effects of early severe deprivation on cognition persist into early adolescence? Findings from the English and Romanian Adoptees Study. Child Development, 77, 696-711. http://dx.doi. org/10.1111/j.1467-8624.2006.00898.x

2. Brodzinsky, D. M. y Pinderhughes, E. (2002). Parenting and child development in adoptive families. En M. Bornstein (Ed.), Handbook of parenting, vol. 1: Children and Parenting (2 $2^{\mathrm{a}}$ Ed.) (pp. 279-327). Hillsdale: Erlbaum.

3. Brown, J. L. y Pollitt, E. (1996). Desnutrición, pobreza y desarrollo intelectual. Investigación y Ciencia, 235, 4-10.

4. Colombo, M., de la Parra, A. y López, I. (1992). Intellec- 
tual and Physical outcome of children undernourished in early life is influenced by later environmental conditions. Developmental Medicine and Child Neurology, 34, 611622. http://dx.doi.org/10.1111/j.1469-8749.1992.tb11492.x

5. Dennis, W. (1973). Children's from the Créche. Nueva York: Appleton Century Crofts.

6. Duyme, M. Dumaret, A. C. y Tomkiewicz, S. (1999) How can be boost IQs of dull children? A late adoption study. Proceedings of the National Academy of Sciences USA, 96, 8790-8794.

7. Fernández, M. y Fuentes, M. J. (2001). Variables infantiles de riesgo en el proceso de adaptación de niños/as de adopciones especiales. Infancia y Aprendizaje, 24, 341-359.

8. Fuentes, M. J. y Fernández, M. (2001). Variables de riesgo y prevención del fracaso en las adopciones especiales. Pedagogía Social. Revista Interuniversitaria, 6-7, 159-174.

9. González, A.M., Quintana, I., Barajas, C., Linero, M.J., Goicoechea, M.A., Fuentes, M.J., Fernández, M. y De la Morena, M.J. (2001). Medio social y desarrollo del lenguaje: un estudio con niños adoptados. Revista de Psicología General y Aplicada, 54, 515-529.

10. González, A.M., Quintana, I., Fernández, M. y Linero, M.J. (2003). Medio social y rendimiento intelectual. Un estudio con niños adoptados. Iberpsicología: Revista Electrónica de la Federación española de Asociaciones de Psicología, 8,5 .

11. Juffer, F., van Ijzendoorn, M.H. y Palacios, J. (2011). Recuperación de niños y niñas tras su adopción. Infancia y Aprendizaje, 34, 3-18. http://dx.doi. org/10.1174/021037011794390102

12. Kreppner, J.M., Rutter, M., Beckett, C., Castle, J., Colvert, E., Groothues, C., Hawkins, A., O’Connor, T.G., Stevens, S. y Sonuga-Barke. E. J. S. (2007). Normality and impairment following profound early institutional deprivation: a longitudinal follow-up into early adolescence. Developmental Psychology, 43, 931-946. http://dx.doi.org/10.1037/00121649.43.4.931

13. Lemos, S. (2003). La psicopatología de la infancia y la adolescencia: consideraciones básicas para su estudio. Papeles del Psicólogo, 85, 19-28.

14. Luthar, S. S. (2006). Resilience in development: A synthesis of research across five decades. En D. Cicchetti y D.J. Cohen (Eds.), Developmental Psychopathology: Risk, disorder, and adaptation (2 $\left.{ }^{\mathrm{a}} \mathrm{Ed}\right)$. Vol 3. (pp. 739-795). Nueva York: Wiley.

15. Luthar, S. S., Cicchetti, D. y Becker, B. (2000). The construct of resilience: A critical evaluation and guidelines for future work. Child Development, 71, 543-562. http:// dx.doi.org/10.1111/1467-8624.00164

16. Masten, A. S. (2001). Ordinary Magic. Resilience processes in development. American Psychologist, 56, 227-238. http://dx.doi.org/10.1037/0003-066X.56.3.227

17. O'Connor, T. G., Rutter, M., Beckett, C., Keaveney, L. y
Kreppner, J. M. (2000). The effects of global severe privation on cognitive competence: Extension and longitudinal follow-up. Child Development, 71, 376-390. http://dx.doi. org/10.1111/1467-8624.00151

18. Palacios, J. (1998). Familias adoptivas. En M. J. Rodrigo y J. Palacios (Coords.), Familia y desarrollo humano (pp. 353-371). Madrid: Alianza.

19. Palacios, J. (1999). Psicología evolutiva: concepto, enfoques, controversias y métodos. En J. Palacios, A. Marchesi, y C. Coll (Comps.), Desarrollo psicológico y educación 1. Psicología evolutiva (pp. 23-78). Madrid: Alianza.

20. Palacios, J. (2007). Después de la adopción: necesidades y niveles de apoyo. Anuario de Psicología, 38, 181-198.

21. Palacios, J. y Sánchez, Y. (1996). Niños adoptados y no adoptados: un estudio comparativo. Anuario de Psicología, 71, 63-85.

22. Palacios, J., Sánchez, Y. y León, E. (2005). Adopción internacional en España: Un nuevo país, una nueva vida. Madrid: Ministerio de Trabajo y Asuntos Sociales.

23. Palacios, J., Sánchez, Y. y Sánchez, E. (1997). La adopción en Andalucía. Sevilla: Consejería de Asuntos Sociales, Junta de Andalucía.

24. Pollit, E. (1987). A critical view of three decades of research on the effects of cronic energy malnutrition on behavioral development. En B. Schurch y N.S. Scrimshaw (Eds.), Chronic Energy Deficiency: Consequences and Related Issues (pp. 77-94). Lausanne: Nestlé Foundation.

25. Ramey, C. T. y Ramey, S. L. (1998). Early intervention and early experience. American Psychologist, 53, 109-120. http://dx.doi.org/10.1037/0003-066X.53.2.109

26. Román, M. (2004). Niños y niñas rumanos procedentes de adopción internacional: ¿son diferentes a los demás? Apuntes de Psicología, 22, 391-402.

27. Roy, P., Rutter, M. y Pickles, A. (2000). Institutional care: Risk from family background or pattern of rearing? Journal of Child Psychology and Psychiatry, 41, 139-149. http:// dx.doi.org/10.1111/1469-7610.00555

28. Rutter, M. (2000). Resilience reconsidered: Conceptual considerations, empirical findings, and policy implications. En J. P. Shonkoff y S.J. Meisels (Eds.), Handbook of early childhood intervention ( $2^{\mathrm{a}}$ Ed.) (pp. 651-682). Nueva York: Cambridge University Press.

29. Rutter, M., O'Connor, T., Beckett, C., Castle, J., Croft, C., Dunn, J., Groothues, C. y Kreppner, J. (2002). Recuperación y déficit tras privaciones iniciales profundas. Bienestar y Protección Infantil, 1, 11-32.

30. Rutter, M. y The English and Romanian Adoptees Study Team (1998). Developmental catch-up, and deficit, following adoption after severe global early privation. Journal of Child Psychology and Psychiatry, 39, 465-476. http://dx.doi.org/10.1111/1469-7610.00343

31. Sameroff, A. J. (1998). Environmental Risk factors in infancy. Pediatrics, 102, 1287-1292. 
32. Sameroff, A. J., Seifer, R., Baldwin, A. y Baldwin, C. (1993). Stability of intelligence from preschool to adolescence: the influence of social and family risk factors. Child Development, 64, 80-97. http://dx.doi. org/10.1111/j.1467-8624.1993.tb02896.x

33. Sayegh, Y. y Dennis, W. (1965). The effect of supplementary experiences upon the behavioral development of infants in institutions. Child Development, 36, 81-90. http:// dx.doi.org/10.2307/1126782

34. Vera, B., Carbelo, B. y Vecina, M. L. (2006). La experiencia traumática desde la psicología positiva: resiliencia y crecimiento postraumático. Papeles del Psicólogo, 27, 40-49.

Fecha de recepción: 20 de mayo de 2011 Fecha de recepción de la versión modificada: 18 de enero de 2012

Fecha de aceptación: 2 de febrero de 2012 